\title{
Nanosizing of Poorly Water Soluble Compounds Using Rotation/Revolution Mixer
}

\author{
Takayuki Takatsuka, ${ }^{a}$ Tomoko Endo, ${ }^{a}$ Yao Jianguo, ${ }^{a}$ Kayo Yuminoki, ${ }^{b}$ and Naofumi Hashimoto $*, b$ \\ ${ }^{a}$ Thinky Corporation; 3-21-5 Kandasamkuma-cho, Chiyoda-ku, Tokyo 101-0025, Japan: and ${ }^{b}$ Setsunan University, 45-1, \\ Nagatoge-cho, Hirakata, Osaka 573-0101, Japan. \\ Received March 16, 2009; accepted July 21, 2009; published online July 28, 2009
}

In this study, nanoparticles of various poorly water soluble compounds were prepared by wet milling that was carried out using a rotation/revolution mixer and zirconia balls. To be compared with Beads mill, rotation/revolution mixer has superior in very quick process $(5 \mathrm{~min})$ and needs very few amounts of zirconia balls (2.4 g) for pulverizing drugs to nanometer range. Phenytoin, indomethacin, nifedipine, danazol, and naproxen were selected as the standard poorly water soluble compounds. Various parameters of the rotation/revolution mixer were studied to decide the optimal pulverization conditions for the production of nanoparticles of the abovementioned compounds. The rotation/revolution speed, shape of the mixing vessel, amount of zirconia balls, and volume of the vehicle (methylcellulose solution) mainly affected the pulverization of the compounds. Using the mixer, phenytoin could be pulverized to nanoparticles within a few minutes. The particle size was confirmed by using a scanning electron microscope and a particle size analyzer. The crystallinity of the pulverized phenytoin particles was confirmed by X-ray diffractometry (XRD) and differential scanning calorimetry (DSC). It was observed that the pulverized phenytoin particles retained their crystallinity, and amorphous phenytoin was not detected. Particles of other poorly water soluble compounds were also reduced to the nanometer range by using this method.

Key words nanoparticle; revolution; mixer; poorly water soluble compound; milling; particle size

Synthesis of water soluble compounds with high pharmacological activity is becoming increasingly difficult. Pulverization is one of the methods that can be used to enhance the dissolution rate of poorly water soluble compounds and improve their bioavailability. ${ }^{1)}$ Pulverization is an important process, especially during the discovery, clinical, and developmental stages of a drug to salvage from the dropout of compound.

Phenytoin is a highly crystalline compound with a high melting point of $295-298^{\circ} \mathrm{C}$ and poor solubility in water. Because of its poor solubility in water, there are very few cases where the concentration of phenytoin in blood reaches the desired levels when administered at a conventional dosage. ${ }^{2,3)}$ According to a few reports, however, the dissolution rate of phenytoin was improved by reducing its particle size, thereby increasing its concentration in blood to the desired level. ${ }^{4-6)}$ Reducing the particle size makes the increase of surface area and as a result of it, dissolution rate of drug increases according to the Nernst-Brunner and Levich modification of the Noyes-Whitney model of dissolution. ${ }^{7,8)}$ Solubility problem could be solved by reducing particle size to nanometer range. ${ }^{9)}$

There are different techniques for pulverizing compounds to obtain nanoparticles-wet-grinding in agitated media milling, stirred media milling, wet co-grinding. ${ }^{10,11)}$ However, in most cases, these techniques are difficult to be carried out. Wet milling is one of the various methods used to obtain nanoparticles of a given compound. The use of the wet mill, NanoCrystal, brought about a remarkable improvement in bioavailability by reducing the size of the particles to the nanometer range. ${ }^{12,13)}$ Conventional beads milling is timeconsuming and requires a large amount of beads for pulverizing a given compound in the grinding chamber. Moreover, troublesome tasks such as the cleaning of balls and machine have to be carried out after the pulverization. The rota- tion/revolution mixer is used to homogenize materials such as epoxy resins, silicone resins, acrylic resins, metal pastes, inks, cosmetics, pastes, and foodstuff. In the pharmaceutical field, beads milling is used for preparing oral formulations for preclinical safety studies. ${ }^{14)}$ Beads milling helps improve product quality and reduce its manufacturing process. Rotation/revolution mixer does not need much amount of compounds and can pulverize a small amount of them (mg order). It can easily scale up from very small volume to large volume. This paper reports on a method to reduce the size of the particles to the nanometer range by using a rotation/revolution mixer and zirconia balls.

\section{Experimental}

Manufacturing Instruments A rotation/revolution mixer (Nano Pulverizer NP-100, Thinky Co., Ltd., Tokyo, Japan) was used to pulverize the compounds. Two types of vessels made of polyacetal were used-one was convex and the other was circular, as shown in Figs. 3a, b. Zirconia (zirconium oxide) balls with diameters of $0.05 \mathrm{~mm}, 0.1 \mathrm{~mm}, 0.3 \mathrm{~mm}, 0.5 \mathrm{~mm}$, and $1 \mathrm{~mm}$ (YTZ-0.05, $-0.1,-0.3,-0.5$, and -0.1 ) were purchased from Nikkato Co., Ltd. (Osaka, Japan).

Materials Poorly water soluble drugs (phenytoin, indomethacin, nifedipine, and naproxen) were purchased from Wako Pure Chemical Co., Ltd. (Osaka, Japan), and Danazol was purchased from Shin Nippon Yakugyo Co., Ltd. (Tokyo, Japan). Methylcellulose (MC) (Metolose SM-4000) and Tween 80 (polysorbate 80 ) were purchased from Shin-Etsu Co., Ltd. (Tokyo, Japan) and Nikko Chemicals Co., Ltd. (Tokyo, Japan), respectively.

Preparation of Suspension The conditions for wet milling, such as the diameter and amount of zirconia balls, revolution speed, running time, and the volume and concentration $(\%, \mathrm{w} / \mathrm{v})$ of aqueous $\mathrm{MC}$ solution, were studied in order to determine optimal conditions for the pulverization of phenytoin. The compound $(100 \mathrm{mg})$ was weighed into the vessel of the mixer. Zirconia balls and an appropriate volume of aqueous MC solution were added to the vessel. Two cases with different running times and volumes of the aqueous MC solution were employed-Case-1, one step of $2000 \mathrm{rpm}$ for $2 \mathrm{~min}$ with $0.6 \mathrm{ml}$ of aqueous MC solution; Case-2, one step of $2000 \mathrm{rpm}$ for $2 \mathrm{~min}$ with $0.6 \mathrm{ml}$ of aqueous MC solution and one step of $2000 \mathrm{rpm}$ for $2 \mathrm{~min}$ with $1 \mathrm{ml}$ of aqueous MC solution. The pulverization conditions were adjusted in accordance with Case- 1 on the basis of the particle size $\left(\mathrm{D}_{50}\right)$ of the pulverized phenytoin. For reference, the original bulk of phenytoin was 
dispersed in $10.0 \mathrm{ml}$ of $0.3 \%$ aqueous MC solution. Freeze-dried samples of the reference and pulverized suspensions were studied for analysis of the various characteristics described in the subsequent paragraphs. Other poorly water soluble drugs (indomethacin, nifedipine, danazol, and naproxen; $100 \mathrm{mg}$ ) were pulverized under the conditions described for Case-2. $0.3 \%$ aqueous MC solution was used as the standard vehicle.

Morphology and Particle Size Distribution The morphology and particle size of the crystals were observed using an SU-1500 scanning electron microscope (SEM) (Hitachi High-Technologies Co., Ltd., Tokyo, Japan). Freeze-dried samples of the reference and pulverized suspensions were used for the SEM analysis. The reference and pulverized suspensions were diluted twenty-folds with water and sonicated for $10 \mathrm{~min}$. Tween 80 was added to the suspension to prevent aggregation, and the final concentration of Tween 80 was $0.1 \%(\mathrm{v} / \mathrm{v})$. The samples were analyzed by a laser diffractometer (Mastersizer 2000, Malvern Instruments, England) with a small-volume dispersing unit (Hydro $2000 \mu \mathrm{P}$, Malvern Instruments). The equivalent volume moment mean diameter D [4,3], and the diameters at $10 \%, 50 \%$, and $90 \%$ of the population distribution $\left(\mathrm{D}_{10}, \mathrm{D}_{50}, \mathrm{D}_{90}\right.$, respectively) were represented as mean particle size and size distribution.

Homogeneity of Suspension The suspension was assayed to assess its homogeneity. The pulverized suspension in the vessel was poured into a 20$\mathrm{ml}$ vial and aliquots of the suspension were periodically collected from the upper, middle, and lower parts of the vial. The concentration of the samples was measured using a SHIMADZU liquid chromatography system (LC20AD with PDA detector, SHIMADZU) with a reverse-phase column $(4.6 \times 150 \mathrm{~mm} ; 5.0 \mu \mathrm{m}$; MILLIPORE). Phenytoin was detected at a UV absorbance of $254 \mathrm{~nm}$. The mobile phase used was $55 \% \mathrm{MeCN} / 45 \%$ water $(\mathrm{v} / \mathrm{v})$, and the flow rate was $0.5 \mathrm{ml} / \mathrm{min}$. Phenytoin was eluted in $4.5 \mathrm{~min}$. The homogeneity of the suspension was indicated by the difference between the mean concentration (\%) of the upper, middle, and lower parts of the vial and the initial concentration of the suspension.

Solid State Characterization by X-Ray Diffraction (XRD) X-ray diffraction was performed using a powder diffractometer (RotaFlex, Rigaku, Japan) with a $\mathrm{Cu} K \alpha$ radiation source at a $40-\mathrm{kV}$ voltage and $30-\mathrm{mA}$ current. Measurements were performed at a scanning speed of $2 \%$ min over a $2 \theta$ range of $5-45^{\circ}$.

Differential Scanning Calorimetry (DSC) Thermal analysis was carried out using a differential scanning calorimeter (DSC Q100, TA instruments). The original bulk and the freeze-dried sample of the pulverized suspension of phenytoin were analyzed at a heating rate of $5{ }^{\circ} \mathrm{C} / \mathrm{min}$ and a temperature range of $30-350^{\circ} \mathrm{C}$.

\section{Results and Discussion}

The rotation/revolution mixer can be used to mix and defoam materials homogeneously without the need for propeller-type agitators. The material is mixed in the vessel by the simultaneous rotation and revolution of the vessel. Zirconia balls with a powerful centrifugal force of $400 \mathrm{G}$ pulverize the materials in a short time.

Theoretical Optimum Ratio of Rotation and Revolution Speeds The efficiency of pulverization of a compound is related to the collision energy of the ball in the vessel. The ball continuously rotates from A to D due to the rotation of the vessel. The ball rises due to the centrifugal force $(\omega)$ of rotation and falls due to the centrifugal force of revolution (Fig. 1).

The ball collides with the particles of the compound on the walls of the vessel, thereby pulverizing the particles. The higher the ball rises in the vessel, the larger is the increase in its collision energy. The proportional force at the beginning of the ball's fall is defined as

$$
F_{\Omega} \cos 45^{\circ} \sin \theta=F_{\omega}
$$

where $F_{\Omega}$ is the centrifugal force of rotation, and $F_{\omega}$ is the centrifugal force of revolution. As the vessel is inclined at an angle of $45^{\circ}$, the centrifugal forces are described as (Fig. 2):

$$
F_{\Omega}=m \Omega^{2}\left(R-r \sin \theta \cos 45^{\circ}\right), \text { and } F_{\omega}=m \omega^{2} r
$$

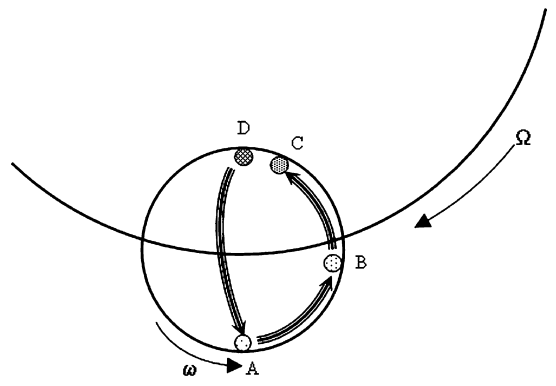

Fig. 1. Movement of a Ball in the Vessel

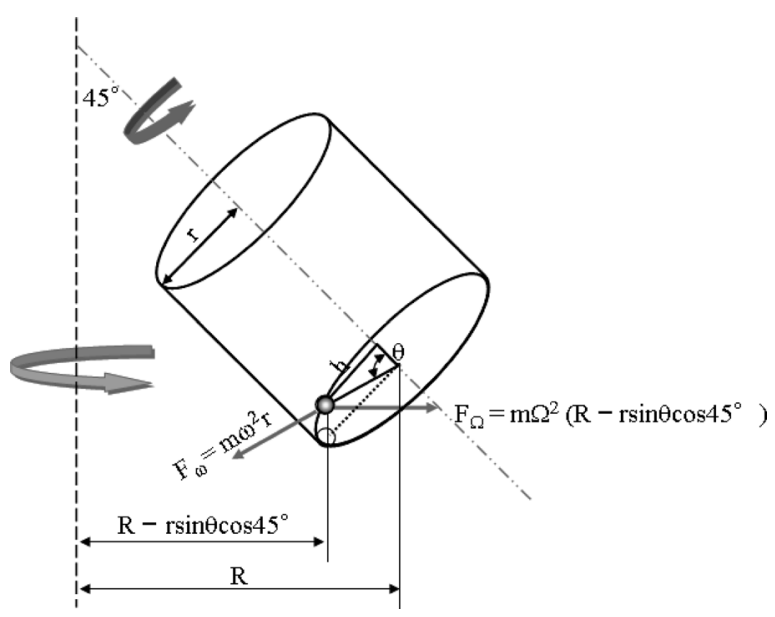

Fig. 2. Relation between Potential Energy and Rotation/Revolution Speed

thus it can be obtained,

$$
\frac{1}{\sqrt{2}} m \Omega^{2}\left(R-\frac{r}{\sqrt{2}} \sin \theta\right) \sin \theta=m \omega^{2} r
$$

where $\omega$ is the rotation speed, $r$ is the radius of rotation and the inside of the vessel, $\Omega$ is the revolution speed, $R$ is the radius of revolution.

Regarding the $h$ as the vertical distance of the ball from the center of the vessel,

$$
\sin \theta=h / r, \quad h=\frac{R}{\sqrt{2}}-\sqrt{\frac{R^{2}}{2}-\frac{\omega^{2}}{\Omega^{2}} \cdot 2 r^{2}}
$$

As the collision energy is related to $h$, the ball at the longest distance position generates the highest collision energy. When $h$ becomes equal to the radius of rotation, the efficiency of pulverization is maximized.

$$
h=r=\frac{R}{\sqrt{2}}-\sqrt{\frac{R^{2}}{2}-\frac{\omega^{2}}{\Omega^{2}} \cdot 2 r}, \frac{\omega}{\Omega}=\sqrt{\frac{1}{\sqrt{2}} \cdot \frac{R}{r}-\frac{1}{2}}
$$

$R$ is $95 \mathrm{~mm}$, and $r$ is $35 \mathrm{~mm}$.

$$
\frac{\omega}{\Omega}=1.19
$$

NP-100 was customized for pulverization, and the rotation speed was set at $2000 \mathrm{rpm}$ for obtaining the optimum revolution/rotation ratio of $1: 1$.

Examination of the Optimized Shape of the Vessel The shape of the vessel was expected to influence the move- 


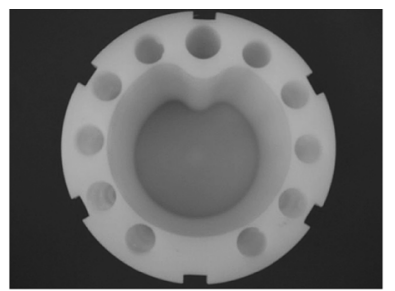

(a)

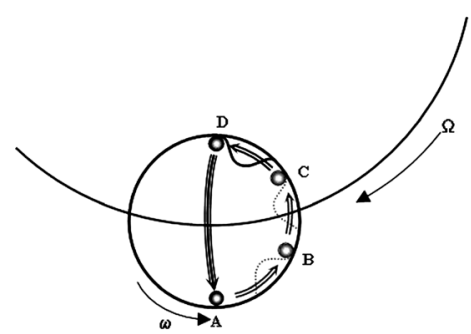

(c)

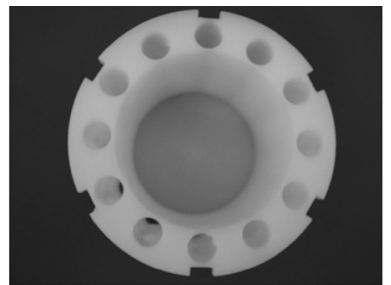

(b)

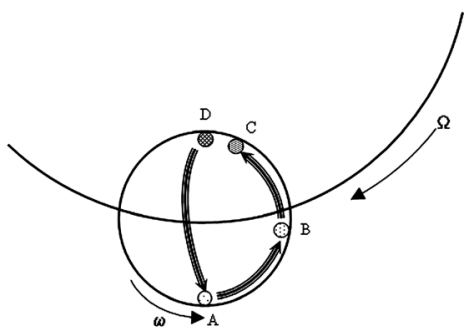

(d)

Fig. 3. The Theoretical Model of the Two Types of Vessels: (a) Convex Vessel, (b) Circular Vessel and (c) Movement of the Ball in the Convex Vessel, (d) Movement of the Ball in the Circular Vessel

ment of the ball during the pulverization of the compound. Two types of vessels (convex and circular) were used, as shown in Figs. 3a and b. By using a convex vessel, the balls, compound, and vehicle are forcibly raised to the highest position (D) and dropped to the bottom (A) (Fig. 3c). The efficiency of pulverization was significantly lesser when using the circular vessel than when using the convex vessel because the number of balls that reached the highest position (D) was less in the circular vessel than in the convex vessel (Fig. 3d). These movements can be seen by using a stroboscope. Phenytoin was used as a model for poorly water soluble compounds. Table 1 shows the differences in $\mathrm{D}_{10}, \mathrm{D}_{50}$, and $\mathrm{D}_{90}$ between the two vessels. Although there were no significant differences in the values of $D_{10}$ and $D_{50}$ between the two vessels, $D_{90}$ was found to be larger in the case of the circular vessel than in the case of the convex vessel. The aggregates of the particles might not have been sufficiently broken in the case of the circular vessel, which had no projections against which the aggregates could collide, unlike in the convex vessel.

Preliminary Examination of the Pulverization Process Preliminary examination of the pulverization process was conducted for the following cases-Case-1 (2000 rpm for $2 \mathrm{~min}$ with $0.6 \mathrm{ml}$ of aqueous MC solution) and Case-2 ( $2000 \mathrm{rpm}$ for $2 \mathrm{~min}$ with $0.6 \mathrm{ml}$ of aqueous MC solution and $2000 \mathrm{rpm}$ for $2 \mathrm{~min}$ with $1 \mathrm{ml}$ aqueous MC solution); this examination was conducted according to the method mentioned in a previous report. ${ }^{14)}$ Most of the particles of phenytoin were pulverized to obtain nanoparticles by using the conditions of Case-1 $\left(\mathrm{D}_{50}: 10.5 \mu \mathrm{m}\right.$ of original bulk $\left.\rightarrow 0.18 \mu \mathrm{m}\right)$, but a small amount of aggregates seemed to exist at particle sizes in the micrometer range (Fig. 4). The distribution of the particle sizes in the micrometer range disappeared by using the conditions of Case-2. The aggregates seemed to be broken down by the additional pulverization in the second step of Case-2 $\left(\mathrm{D}_{90}: 3.55 \mu \mathrm{m}\right.$ of Case- $\left.1 \rightarrow 0.27 \mu \mathrm{m}\right)$ (Table 2).

On the basis of results of these experiments, the conditions used in Case-1 were employed in the following studies as they were considered to be the optimal conditions for the
Table 1. Comparison between the Particle Size Distributions Obtained Using the Convex and the Circular Vessels after Pulverization

\begin{tabular}{lccc}
\hline \hline \multirow{2}{*}{ Vessel } & \multicolumn{3}{c}{ Particle size $(\mu \mathrm{m})$} \\
\cline { 2 - 4 } & $\mathrm{D}_{10}$ & $\mathrm{D}_{50}$ & $\mathrm{D}_{90}$ \\
\hline Circular & 0.079 & 0.197 & 3.032 \\
Convex & 0.067 & 0.123 & 0.239 \\
\hline
\end{tabular}

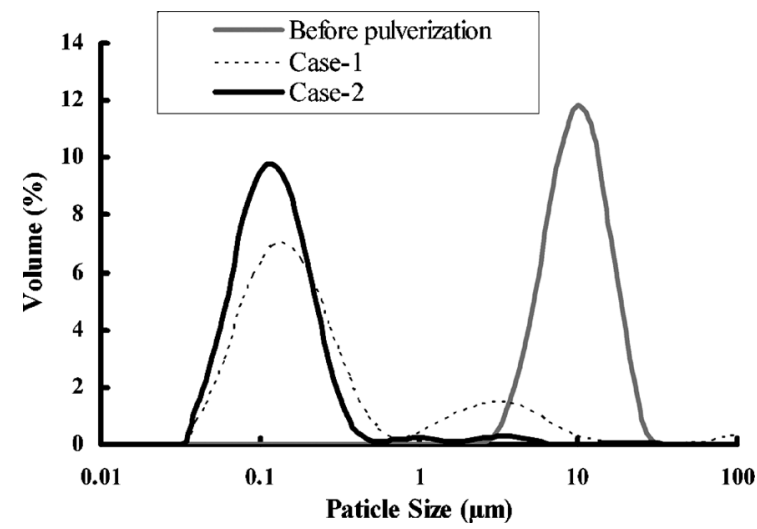

Fig. 4. Particle Size Distribution of Pulverized Phenytoin under the Conditions Used in Case-1 and Case-2

Case-1: $2000 \mathrm{rpm}$ for $2 \mathrm{~min}$ with $0.6 \mathrm{ml}$ aqueous MC solution, Case-2: $2000 \mathrm{rpm}$ for $2 \mathrm{~min}$ with $0.6 \mathrm{ml}$ aqueous MC solution and $2000 \mathrm{rpm}$ for $2 \mathrm{~min}$ with $1 \mathrm{ml}$ aqueous MC solution.

Table 2. Particle Size Distribution of Phenytoin before Pulverization, after Pulverization Using the Conditions in Case-1, and after Pulverization Using the Conditions in Case-2

\begin{tabular}{ccccc}
\hline \hline \multirow{2}{*}{ Step times } & \multicolumn{4}{c}{ Particle size $(\mu \mathrm{m})$} \\
\cline { 2 - 5 } & $\mathrm{D}[4,3]$ & $\mathrm{D}_{10}$ & $\mathrm{D}_{50}$ & $\mathrm{D}_{90}$ \\
\hline Before & 11.4 & 5.79 & 10.5 & 18.4 \\
Case-1 & 3.07 & 0.08 & 0.18 & 3.55 \\
Case-2 & 0.29 & 0.06 & 0.13 & 0.27 \\
\hline
\end{tabular}


pulverization of phenytoin to nanoparticles, and $\mathrm{D}_{50}$ was used as the reference parameter for the particle size obtained by pulverization under the aforementioned conditions. However, the conditions used in Case- 2 would be necessary in order to prevent the aggregation of the phenytoin particles for the practical use.

Determining the Optimal Conditions for the Rotation/Revolution of the Mixer The optimal conditions for the wet milling of phenytoin were studied by taking the following parameters into consideration (Fig. 5): the diameter and amount of zirconia balls, revolution speed and running time, and volume and concentration of the aqueous MC solution. These conditions were also applied for the pulverization of other poorly water soluble compounds.

Diameter of Zirconia Ball The diameter of the zirconia ball was changed from 0.05 to $1 \mathrm{~mm}$ (Fig. 5a). Phenytoin could be pulverized to obtain nanoparticles using zirconia balls with diameters in the range of $0.1-0.5 \mathrm{~mm}$. Pulverized particles with the smallest diameters $\left(D_{50}=171 \mathrm{~nm}\right)$ were obtained using zirconia balls of $0.1 \mathrm{~mm}$ diameter. $\mathrm{D}_{50}$ seemed to increase by the use of smaller as well as larger zirconia balls $(0.05 \mathrm{~mm}, 1 \mathrm{~mm})$. This phenomenon was understood by the collision energy equation ${ }^{15)}$ as given below:

$$
E_{W}=\sum_{j=1}^{n} \frac{1}{2 W} m v_{j}^{2}
$$

Here, $E_{W}$ is the collision energy, $W$ is the weight of the compound, $m$ is the weight of the zirconia ball, $v$ is the relative speed of the zirconia ball and the particles of the compound, and $n$ is the number of collisions between the ball and the particles of the compound. The smaller the diameter of the zirconia ball, the larger is its total surface area; thus, the number of collisions $(n)$ increases. Since $m$ is proportional to the diameter of the zirconia balls, the weight of the smaller zirconia balls was not sufficient to pulverize the particles of the compound. On the other hand, with larger zirconia balls, $n$ was not sufficiently high. Therefore, the optimum diameter of the zirconia balls was determined to be $0.1 \mathrm{~mm}$.

Amount of Zirconia Ball The amount of zirconia balls with a diameter of $0.1 \mathrm{~mm}$ was changed from 0.3 to $4.8 \mathrm{~g}$ (Fig. 5b). Phenytoin could be pulverized to obtain nanoparticles with $0.3-4.8 \mathrm{~g}$ of zirconia balls. The efficiency of pulverization was maximum $\left(D_{50}=143 \mathrm{~nm}\right)$ when the amount of zirconia balls was $2.4 \mathrm{~g}$. When a large amount of zirconia balls was used, the balls interfered with each other and caused a decrease in the collision energy. On the one hand, the conventional beads mill requires a large amount of beads, while on the other hand, the rotation/revolution mixer could pulverize the compound with a small amount of zirconia balls. There was a large difference between the amount of balls required in the rotation/revolution mixer and the amount of beads required in the conventional beads mill.

Revolution Speed The revolution speed was increased from 600 to $2000 \mathrm{rpm}$ while maintaining the rotation/revolution ratio at $1: 1$ (Fig. 5c). Phenytoin could be pulverized to obtain nanoparticles at revolution speeds of 1000 $2000 \mathrm{rpm}$, and the smallest particles $\left(\mathrm{D}_{50}=127 \mathrm{~nm}\right)$ were obtained when the revolution speed was maximum (2000 rpm). The revolution speed is related to the speed of collision between the zirconia balls and the particles of the compound. Therefore, revolution at the maximum speed was expected to be the most effective condition for the pulverization. At a revolution speed of $800 \mathrm{rpm}$, phenytoin was not efficiently pulverized as $v$ was not sufficiently high.

Running Time (for the Pulverization) The running time was varied from $15 \mathrm{~s}$ to $4 \mathrm{~min}$ (Fig. 5d). Although phenytoin could be pulverized to obtain nanoparticles at all the running times in this range, particles that were not sufficiently pulverized remained in the suspension when the run-

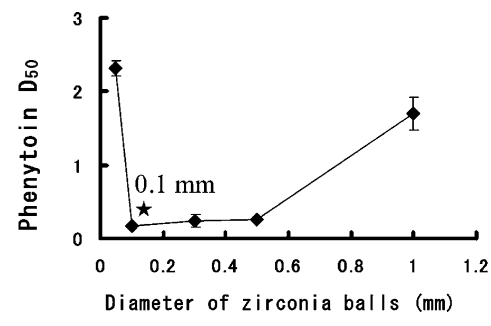

(a)

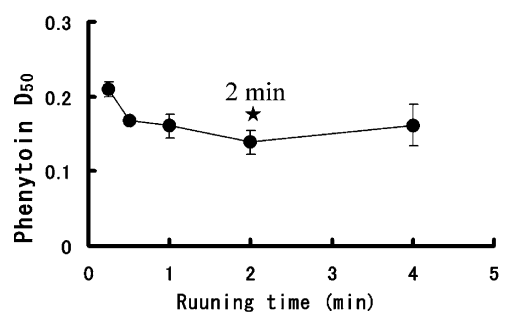

(d)

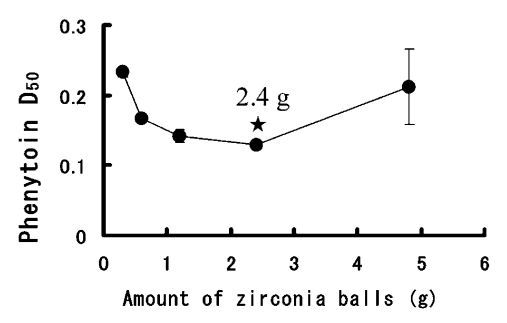

(b)

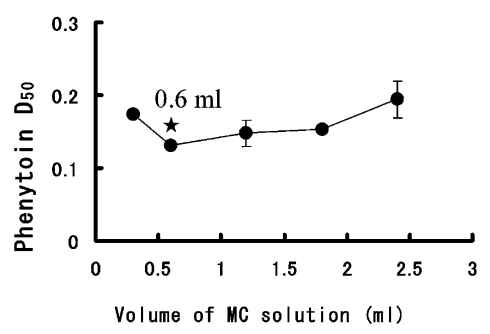

(e)

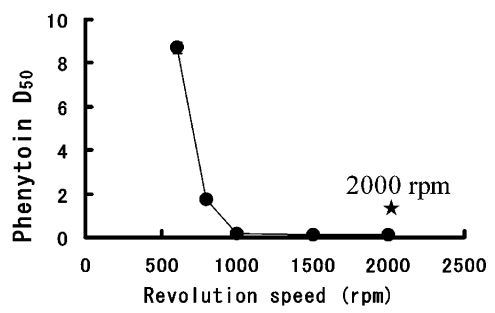

(c)

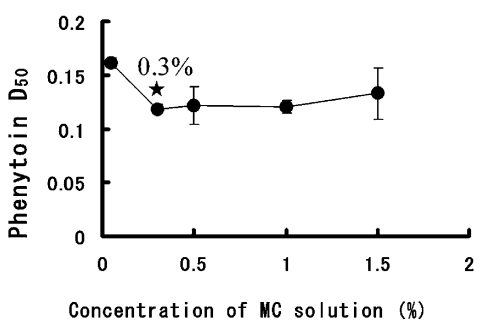

(f)

Fig. 5. Examination of the Conditions of Rotation/Revolution Mixer: (a) Diameter of Zirconia Balls, (b) Amount of Zirconia Balls, (c) Revolution Speed, (d) Running Time, (e) Volume of MC Solution, and (f) Concentration of MC Solution

These experiments were conducted three times, and the average of $\mathrm{D}_{50}$ were calculated. The vertical bars show the distribution of $\mathrm{D}_{50}$ values, and the asterisk indicates the smallest particle size. 


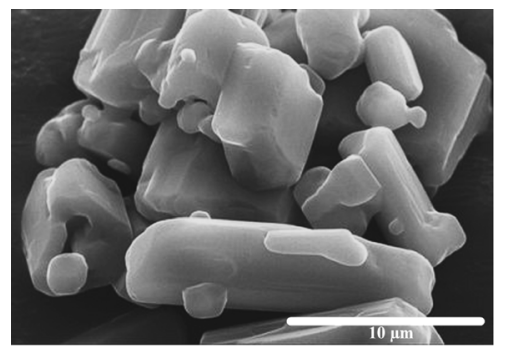

(a)

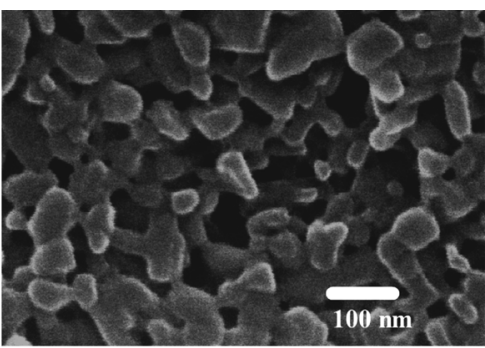

(b)

Fig. 6. SEM Micrographs of Phenytoin: (a) Original Bulk, (b) Pulverized Phenytoin Bulk

ning time was in the range of $15 \mathrm{~s}$ to $1 \mathrm{~min}$. The smallest particles $\left(D_{50}=138 \mathrm{~nm}, D_{90}=455 \mathrm{~nm}\right)$ were obtained at a running time of $2 \mathrm{~min}$. However, a longer running time (4 min) caused overheating, and the particles of phenytoin dried out and tended to aggregate $\left(\mathrm{D}_{50}=162 \mathrm{~nm}, \mathrm{D}_{90}=4.11 \mu \mathrm{m}\right)$.

Volume of $\mathbf{0 . 3 \%}$ Aqueous MC Solution The volume of the vehicle used in the first step in Case-1 was varied from 0.3 to $2.4 \mathrm{ml}$ (Fig. 5e). Phenytoin could be pulverized to obtain nanoparticles at all the volumes in this range, and the smallest particles $\left(D_{50}=131 \mathrm{~nm}\right)$ were obtained at a volume of $0.6 \mathrm{ml}$. As the volume of the vehicle increases, $n$ decreases. Therefore, the particles were not sufficiently pulverized at volumes greater than $0.6 \mathrm{ml}$.

Concentration of MC Solution The MC concentration in the vehicle was varied from 0.05 to $1.5 \%$ (w/v) (Fig. 5f). Phenytoin could be pulverized to obtain nanoparticles at all concentrations in this range, and the smallest particles $\left(\mathrm{D}_{50}=\right.$ $119 \mathrm{~nm}$ ) were obtained at an MC concentration of $0.3 \%$ (w/v). The concentration of the aqueous MC solution did not have a strong influence on the pulverization of phenytoin.

The conditions used in Case- 2 are necessary in order to prevent the aggregation of the phenytoin particles. The effect of the volume of the aqueous MC solution from 1 to $2 \mathrm{ml}$ was examined in the second step in Case-2. This change had not effect on the aggregation of the phenytoin particles. The second step could prevent aggregating phenytoin particles independent of the volume of the aqueous MC. Then, $1 \mathrm{ml}$ of $0.3 \%$ aqueous $\mathrm{MC}$ solution and a running time of $2 \mathrm{~min}$ were set as the parameters for the second step in Case-2. The optimal conditions for the pulverization of phenytoin are summarized in Table 3 as the conditions used in the first step in any cases were thought to play an important role in the pulverization of phenytoin.

Analysis of Phenytoin Particles The freeze-dried powder of phenytoin suspension was used for SEM analysis. The particle sizes of the reference sample (Fig. 6a) and pulverized phenytoin (Fig. 6b) were $11 \mu \mathrm{m}$ and $100-200 \mathrm{~nm}$, respectively. The results of the particle size distribution analysis revealed that $\mathrm{D}_{50}$ changed from $11 \mu \mathrm{m}$ to $129 \mathrm{~nm}$ (Fig. 7) after pulverization. Although freeze drying process has a risk of crystal growth, significant crystal growth was not found by the SEM analysis and the particle size distribution analysis. The loading amount of phenytoin was increased to $1 \mathrm{~g}$ to verify the possibility of scaling up by modifying the optimal conditions ( $2000 \mathrm{rpm}$ for $2 \mathrm{~min}$ with $4.2 \mathrm{ml}$ of aqueous MC solution and $2000 \mathrm{rpm}$ for $2 \mathrm{~min}$ with $4.2 \mathrm{ml}$ aqueous MC solution, $0.1 \mathrm{~mm}$ diameter zirconia ball $16.8 \mathrm{~g}$ ). The same pulverized bulk as the case of $100 \mathrm{mg}$ was obtained as shown in
Table 3. Standard Conditions (Case-2) for the Pulverization of $10 \mathrm{mg} / \mathrm{ml}$ of Phenytoin

\begin{tabular}{|c|c|c|c|}
\hline \multicolumn{2}{|c|}{ Parameters } & 1st step & 2nd step \\
\hline \multicolumn{2}{|c|}{ Revolution speed } & $2000 \mathrm{rpm}$ & $2000 \mathrm{rpm}$ \\
\hline \multicolumn{2}{|c|}{ Running time } & $2 \min$ & $2 \mathrm{~min}$ \\
\hline \multirow[t]{2}{*}{ Vehicle } & Concentration & $0.3 \%$ & - \\
\hline & Volume & $0.6 \mathrm{ml}$ & $1 \mathrm{ml}$ \\
\hline \multirow[t]{2}{*}{ Zirconia ball } & Diameter & $0.1 \mathrm{~mm}$ & - \\
\hline & Amount & $2.4 \mathrm{~g}$ & - \\
\hline
\end{tabular}

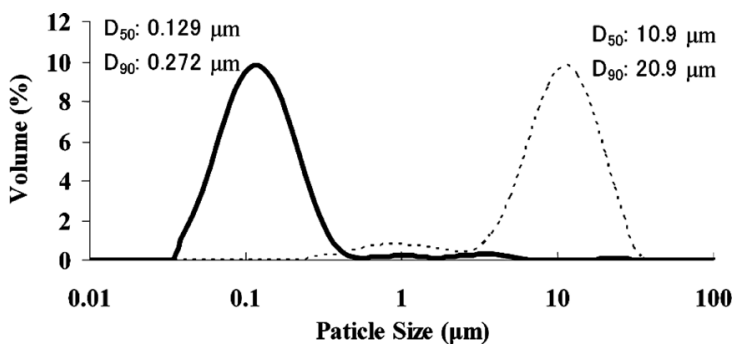

Fig. 7. Particle Size Distribution of the Original (---) and Pulverized Phenytoin Bulk (一)

Table 4. Scaling Up of the Amount of Phenytoin Bulk

\begin{tabular}{ccccc}
\hline \hline \multirow{2}{*}{$\begin{array}{c}\text { Loading } \\
\text { amount of } \\
\text { phenytoin }\end{array}$} & $\mathrm{D}[4,3]$ & $\mathrm{D}_{10}$ & $\mathrm{D}_{50}$ & $\mathrm{D}_{90}$ \\
\cline { 2 - 5 } & 0.29 & 0.06 & 0.13 & 0.27 \\
$100 \mathrm{mg}$ & 0.13 & 0.07 & 0.12 & 0.21 \\
\hline $\mathrm{g}$ & & &
\end{tabular}

Table 4.

The freeze-dried sample of the pulverized phenytoin was used for DSC studies. The melting point of the original bulk of phenytoin was $298^{\circ} \mathrm{C}$, whereas the melting point of the freeze-dried sample of the pulverized phenytoin decreased to $284^{\circ} \mathrm{C}$ (Fig. 8). The melting point of pulverized phenytoin was lower than that of the original bulk. For DSC studies, phenytoin was pulverized with the aqueous MC solution and then dried. The residual sample contained MC. Subsequently, the inclusion of $\mathrm{MC}$ affected the melting point of phenytoin, and the melting point of the pulverized phenytoin might have been lowered due to a decrease in its eutectic temperature. However, there was not found an exothermal peak which showed the existence of amorphous of phenytoin. These results showed that the pulverized phenytoin did not include a lot of amorphous of phenytoin. 


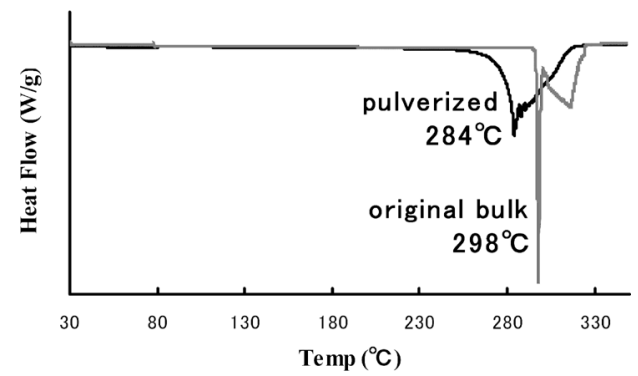

Fig. 8. DSC Thermograph of the Original and Pulverized Phenytoin Bulk
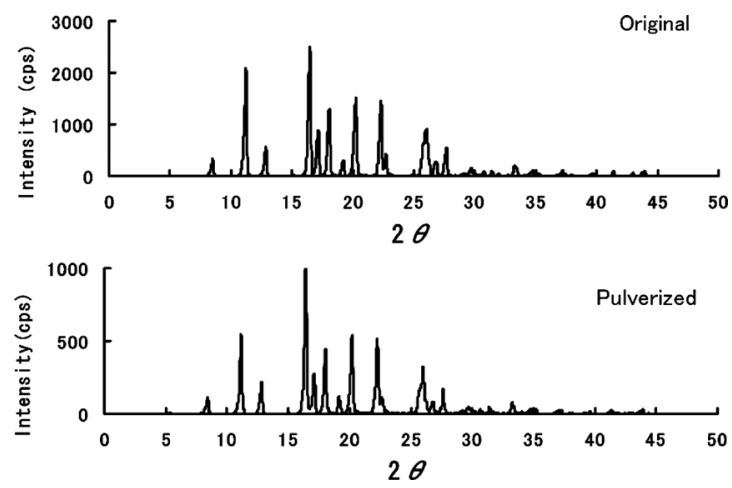

Fig. 9. XRD Patterns of the Original and Pulverized Phenytoin Bulk

Table 5. Mean Concentration of Phenytoin in Various Parts of the Vessel after Pulverization

\begin{tabular}{lcccc}
\hline \hline & $\begin{array}{c}\text { Upper } \\
(\%)\end{array}$ & $\begin{array}{c}\text { Middle } \\
(\%)\end{array}$ & $\begin{array}{c}\text { Lower } \\
(\%)\end{array}$ & $\begin{array}{c}\text { Total average } \\
(\%)\end{array}$ \\
\hline $0 \mathrm{~h}$ & - & - & - & - \\
$0.5 \mathrm{~h}$ & 101.6 & 99.2 & 100.3 & 100.3 \\
$1 \mathrm{~h}$ & 99.8 & 99.0 & 99.9 & 99.6 \\
\hline
\end{tabular}

XRD was performed to investigate the crystallinity of phenytoin (Fig. 9). Figure 9 shows the results of X-ray powder diffraction of the original bulk and the freeze-dried sample of the pulverized phenytoin. The baseline of the freezedried sample was similar to the original bulk and the significant halo peak was not found. These results also showed that the phenytoin particles almost retained their crystallinity after the pulverization.

Pulverization of Other Poorly Water Soluble Compounds Other poorly water soluble compounds (naproxen, nifedipine, danazol, and indomethacin) were pulverized by using the optimal conditions of Case-2 (Fig. 10). Therefore, the use of the rotation/revolution mixer in wet milling was found to be efficient to reduce the particles of poorly water soluble compounds to the nanometer range.

Stability of the Suspension of Phenytoin The average concentration of each aliquot of the suspension, which was periodically collected from the upper, middle, and lower parts of the vessel, was uniform for up to $1 \mathrm{~h}$. Therefore, the nanoparticles in the suspension could be applied in vivo as a homogeneous suspension for up to $1 \mathrm{~h}$, as shown in Table 5.

\section{Conclusion}

The use of the rotation/revolution mixer with zirconia balls had the following advantages:

1) Nanosizing poorly water soluble compounds: Five kinds of poorly water soluble compounds with different physicochemical properties could be pulverized to nanoparticles by using the rotation/revolution mixer.

2) Running time: With the rotation/revolution mixer, particles of a given compound could be effectively pulverized to nanoparticles within a few minutes. The powerful centrifugal force of $400 \mathrm{G}$ could generate sufficient collision energy between the zirconia balls and the particles of the compound.

3) Crystallinity: The results of DSC and XRD indicated that the particles of phenytoin retained their crystallinity

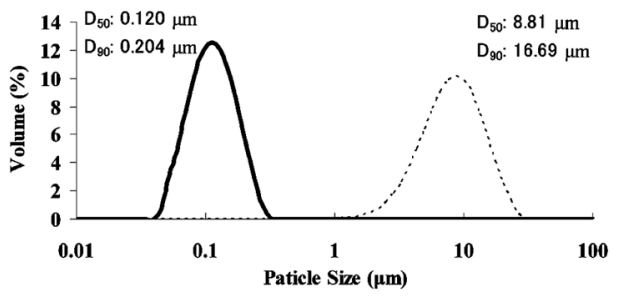

(a) naproxen

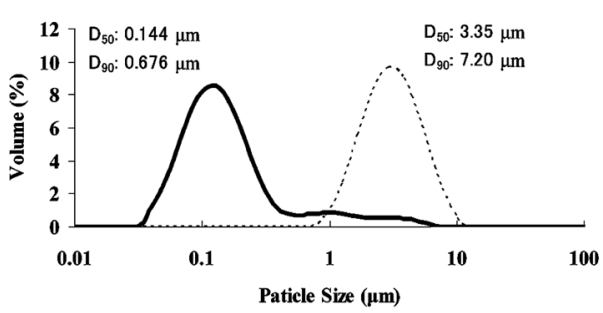

(c) danazol

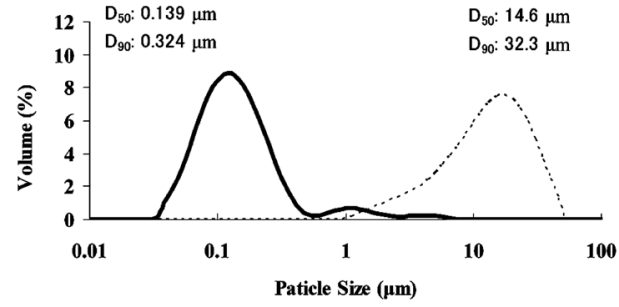

(b) nifedipine

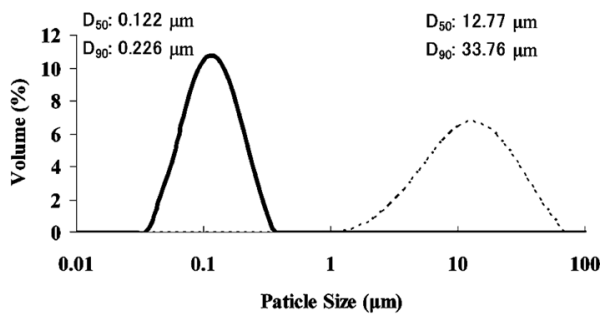

(d) indomethacin

Fig. 10. Particle Size Distribution Profiles of Original (---) and Pulverized (-) Poorly Water Soluble Compounds 
even after the pulverization, and amorphous phenytoin was not detected.

4) Preparation in a closed container: Since the suspension was prepared in a closed container, contamination by external agents was minimized.

In conclusion, a rotation/revolution mixer is well suited for reducing the particle sizes of poorly water soluble compounds to the nanometer range. This method is quite useful for preparing oral suspensions for pharmacological, pharmacokinetic, and safety studies on animals during the drug discovery and preclinical studies. This easy and quick nanosizing method has enough potential to expand a new approach for preparing extemporaneous formulations for the first-inhuman and early clinical studies of various candidate compounds. Compounds whose physicochemical properties are significantly different from the ones used in this study must be studied in order to increase the applications of the rotation/revolution mixer.

\section{References}

1) Yokoyama T., Yakuzaigaku, 68, 209-216 (2008).

2) Shinkuma D., Hashimoto H., Yamanaka Y., Morita Y., Acta Med.
Hyogo, 1, 141-147 (1976).

3) Miyamoto K., Nou to Hattatsu, 6, 444-455 (1976).

4) Unno K., Suto I., Fukui R., Kagaya S., Nakata H., Byoin Yakugaku, 10, 323-330 (1984).

5) Yakou S., Umehara K., Sonobe T., Nagai T., Sugihara M., Fukuyama Y., Chem. Pharm. Bull., 32, 4130-4136 (1984).

6) Glazko A. J., Chang T., "Antiepileptic Drugs," ed. by Woodbury D. M., Penry J. K., Schmidt R. P., Raven Press, New York, 1972, pp. 127136.

7) Dressman J. B., Amidon G. L., Reppas C., Shah V. P., Pharm. Res., 15, 11-22 (1998).

8) Hörter D., Dressman J. B., Adv. Drug Deliv. Rev., 46, 75-87 (2001).

9) Müller R. H., Keck C. M., J. Biotechnol., 113, 151-170 (2004).

10) Mende S., Stenger F., Peukert W., Schwedes J., Powder Technol., 132, 64-73 (2003).

11) Pongpeerapat A., Wanawongthai C., Tozuka Y., Moribe K., Yamamoto K., Int. J. Pharm., 352, 309-316 (2008).

12) Jinno J., Kamada N., Miyake M., Yamada K., Mukai T., Odomi M., Toguchi H., Liversidge G. G., Higaki K., Kimura T., J. Controlled Release, 111, 56-64 (2006).

13) Wu Y., Loper A., Landis E., Hettrick L., Novak L., Lynn K., Chen C., Thompson K., Higgins R., Batra U., Shelukar S., Kwei G., Storey D., Int. J. Pharm., 285, 135-146 (2004).

14) Niwa T., Hashimoto N., Int. J. Pharm., 350, 70-78 (2008).

15) Kano J., Miyazaki M., Saito F., Adv. Powder Technol., 11, 333-342 (2000). 